Nick BEZHANiSHViLI

David Gabelaia

Silvio Ghilardi

MAMUKA JiBLADZE

\title{
Admissible Bases Via Stable Canonical Rules
}

\begin{abstract}
We establish the dichotomy property for stable canonical multi-conclusion rules for IPC, K4, and S4. This yields an alternative proof of existence of explicit bases of admissible rules for these logics.
\end{abstract}

Keywords: Admissible rules, Admissible bases, Modal logic, Intuitionistic logic, Modal algebras, Heyting algebras, Canonical formulas.

\section{Introduction}

An inference rule is admissible in a given logical system $L$ if no new theorems are derived by adding this rule to the rules of inference of $L$. Friedman [10] raised the question whether admissibility of rules in the intuitionistic propositiolculus (IPC) is decidable. A solution to this problem for IPC, as well as for well-known systems of modal logic such as K4 and S4, was first given by Rybakov ([26,27], see also the comprehensive book [24] and the references therein). An alternative solution via projectivity and unification was supplied in $[11,12]$. Explicit bases for admissible rules were built in [15, $17,22,23,25]$. We refer to Goudsmit [14] for a modern historic account of the admissibility problem.

Recently Jeřábek [18] developed a new technique for building bases for admissible rules by generalizing Zakharyaschev's canonical formulas [29] to multi-conclusion canonical rules, and by developing the dichotomy property for canonical rules. This property states that a canonical multi-conclusion rule is either admissible or equivalent to an assumption-free rule. Our goal is to establish the same property for stable multi-conclusion canonical rules for IPC, K4, and S4. These rules were recently introduced in [1], where it was shown that each normal modal multi-conclusion consequence relation is

Presented by Heinrich Wansing; Received July 30, 2015 
axiomatizable by stable multi-conclusion canonical rules. The same result for intuitionistic multi-conclusion consequence relations was established in [2].

The proof methodology we follow is similar to [18] and goes through a semantic characterization of non-admissible stable canonical rules in terms of the finite domains they are built from. In spite of the similarities, the semantic characterization we obtain is different than the one given in [18]. As a simple corollary of our main theorem, similarly to [18], we obtain decidability of the admissibility problem for IPC, K4 and S4. Finally, we note that admissibility for the basic modal logic $\mathbf{K}$ is a long standing open problem. While the proofs of this paper do not directly apply to $\mathbf{K}$, we observe that the method of stable canonical rules, unlike that of canonical rules of [18], is not limited to the transitive case. Therefore, our method is potentially applicable to non-transitive logics such as $\mathbf{K}$.

The paper is organised as follows: In Section 2 we recall Esakia duality for Heyting algebras, multi-conclusion consequence relations and stable canonical rules for IPC. In Section 3 we obtain an explicit basis of admissible rules for IPC via stable canonical rules and prove that the latter have the dichotomy property. In Section 4 we recall duality for modal algebras, modal multi-conclusion consequence relations and stable canonical rules for modal logic. Finally, in Section 5 we obtain explicit bases of admissible rules for $\mathbf{K} 4$ and $\mathbf{S} 4$ via stable canonical rules and prove their dichotomy property.

\section{Preliminaries on Heyting Algebras and IPC}

\subsection{Esakia Duality for Heyting Algebras}

We recall that a Heyting algebra is a bounded distributive lattice with an additional binary operation $\rightarrow$ that is the residual of $\wedge$. For Heyting algebras $A$ and $B$, a Heyting homomorphism is a bounded lattice homomorphism $h: A \rightarrow B$ such that $h(a \rightarrow b)=h(a) \rightarrow h(b)$ for each $a, b \in A$. Let Heyt be the category of Heyting algebras and Heyting homomorphisms. It is well known (see, e.g., [21, Chap. IX] or [6, Chap. 7]) that Heyting algebras provide an adequate algebraic semantics for superintuitionistic logics. In fact, there is a dual isomorphism between the (complete) lattice of superintuitionistic logics and the (complete) lattice of varieties of Heyting algebras.

In order to introduce topological duality for Heyt, we need to fix some notation for posets. If $X$ is a poset (partially ordered set), we denote the partial order on $X$ by $\leqslant$. For $Y \subseteq X$, we recall that the down-set of $Y$ is the set $\downarrow Y=\{x \in X: \exists y \in Y$ with $x \leq y\}$. The up-set of $Y$ is defined dually and is denoted by $\uparrow Y$. If $Y$ is a singleton set $\{y\}$, then we use $\downarrow y$ and $\uparrow y$ 
instead of $\downarrow\{y\}$ and $\uparrow\{y\}$, respectively. We call $U \subseteq X$ an up-set if $x \in U$ and $x \leq y$ imply $y \in U$. A down-set of $X$ is defined dually. For $Y \subseteq X$ we denote by $\max Y$, resp. $\min Y$ the set of its maximal, resp. minimal points. That is, $\max Y=\{y \in Y \mid Y \cap \uparrow y=\{y\}\}$ and $\min Y=\{y \in Y \mid Y \cap \downarrow y=\{y\}\}$.

An Esakia space is a Priestley space $X$ such that $\downarrow U$ is clopen for each clopen $U$ of $X$; recall that a poset $X$ is a Priestley space if $X$ is a compact space and for each $x, y \in X$, from $x \not \leq y$ it follows that there is a clopen (closed and open) up-set $U$ of $X$ such that $x \in U$ and $y \notin U$. It follows easily from e.g. $[8,11.15(\mathrm{i})]$ that for any Priestley space $(X, \leqslant)$, any closed subset $Y \subseteq X$ and any $y \in Y$ there are $y_{1} \in \min Y, y_{2} \in \max Y$ with $y_{1} \leqslant y \leqslant y_{2}$.

For posets $X$ and $Y$, a map $f: X \rightarrow Y$ is order-preserving if $x \leq y$ implies $f(x) \leq f(y)$ for all $x, y \in X$; an order-preserving $f$ is said to be a bounded morphism (or p-morphism) iff for each $x \in X$ and $y \in Y$, from $f(x) \leq y$ it follows that there exists $z \in X$ such that $x \leq z$ and $f(z)=y$.

For Esakia spaces $X$ and $Y$, a map $f$ is an Esakia morphism if it is a bounded morphism which is also continuous. Let Esa be the category of Esakia spaces and Esakia morphisms.

By Esakia duality [9], Heyt is dually equivalent to Esa (the dual of a Heyting algebra $A$ is indicated with $\left.A_{*}\right)$. The functors $(-)_{*}$ : Heyt $\rightarrow$ Esa and $(-)^{*}:$ Esa $\rightarrow$ Heyt that establish this dual equivalence are constructed as follows. For a Heyting algebra $A$, let $A_{*}=(X, \leqslant)$, where $X$ is the space of all prime filters of $A$ (topologized by the subbasis $\{\alpha(a), X \backslash \alpha(a): a \in A\}$, where $\alpha(a)=\{x \in X: a \in x\})$ and $x \leqslant y$ iff $x \subseteq y$. For a Heyting algebra homomorphism $h$, let $h_{*}=h^{-1}$. For an Esakia space $(X, \leqslant)$, let $(X, \leqslant)^{*}=$ $A$, where $A$ is the Heyting algebra of clopen up-sets of $X$, with meet and join given by intersection and union respectively and with implication given by $U \rightarrow V=X \backslash \downarrow(U \backslash V)$. For an Esakia morphism $f$, let $f^{*}=f^{-1}$.

It follows from Esakia duality that onto Heyting homomorphisms dually correspond to 1-1 Esakia morphisms, and 1-1 Heyting homomorphisms to onto Esakia morphisms. In particular, homomorphic images of $A \in$ Heyt correspond to closed up-sets of the Esakia dual of $A$.

\subsection{Intuitionistic Multi-conclusion Consequence Relations}

We use greek letters $\gamma, \delta, \ldots, \varphi, \psi, \ldots$ to denote formulas built up from propositional variables using the connectives $\neg, \wedge, \vee, \rightarrow, \perp, \top$. A valuation on a Heyting algebra $A$ is a map associating an element of $A$ with every propositional variable. It is then extended to all formulas in a standard way. An intuitionistic Kripke model is a triple $(X, \leqslant, V)$ where $(X, \leqslant)$ is a poset and $V$ is a valuation on the Heyting algebra of its up-sets. We use letters 
$\mathfrak{M}, \mathfrak{N}, \ldots$ for Kripke models and the notation $\mathfrak{M}, x \models \varphi$ to mean that $x$ belongs to $V(\varphi)$, where $V$ is the valuation on the Kripke model $\mathfrak{M}$. The notation $\mathfrak{M}=\varphi$ means that $\mathfrak{M}, x \mid=\varphi$ holds for all $x$ from the underlying poset of $\mathfrak{M}$.

A multi-conclusion rule is an expression $\Gamma / \Delta$, where $\Gamma, \Delta$ are finite sets of formulas; if $\Gamma=\left\{\gamma_{1}, \ldots, \gamma_{n}\right\}$ and $\Delta=\left\{\delta_{1}, \ldots, \delta_{m}\right\}$, the rule might be displayed as

$$
\frac{\gamma_{1}, \ldots, \gamma_{n}}{\delta_{1}|\cdots| \delta_{m}}
$$

If $\Delta=\{\varphi\}$, then $\Gamma / \Delta$ is called a single-conclusion rule and is written $\Gamma / \varphi$. If $\Gamma=\varnothing$, then $\Gamma / \Delta$ is called an assumption-free rule and is written $/ \Delta$. Assumption-free single-conclusion rules $/ \varphi$ can be identified with formulas $\varphi$.

DeFinition 2.1. An intuitionistic multi-conclusion consequence relation is a set $\mathcal{S}$ of multiple conclusion rules such that

(1) $\varphi / \varphi \in \mathcal{S}$.

(2) $\varphi, \varphi \rightarrow \psi / \psi \in \mathcal{S}$.

(3) $/ \varphi \in \mathcal{S}$ for each theorem $\varphi$ of IPC (i.e. of intuitionistic propositional calculus).

(4) If $\Gamma / \Delta \in \mathcal{S}$, then $\Gamma, \Gamma^{\prime} / \Delta, \Delta^{\prime} \in \mathcal{S}$.

(5) If $\Gamma / \Delta, \varphi \in \mathcal{S}$ and $\Gamma, \varphi / \Delta \in \mathcal{S}$, then $\Gamma / \Delta \in \mathcal{S}$.

(6) If $\Gamma / \Delta \in \mathcal{S}$ and $\sigma$ is a substitution, then $\sigma(\Gamma) / \sigma(\Delta) \in \mathcal{S}$.

We denote the smallest intuitionistic multi-conclusion consequence relation by $\mathcal{S}_{\text {IPC }}$. For a set $\mathcal{R}$ of multi-conclusion rules, let $\mathcal{S}_{\text {IPC }}+\mathcal{R}$ be the smallest intuitionistic multi-conclusion consequence relation containing $\mathcal{R}$. If $\mathcal{S}=\mathcal{S}_{\text {IPC }}+\mathcal{R}$, then we say that $\mathcal{S}$ is axiomatized by $\mathcal{R}$ or that $\mathcal{R}$ is a basis for $\mathcal{S}$. Whenever $\Gamma / \Delta$ belongs to $\mathcal{S}_{\text {IPC }}+\mathcal{R}$ we say that $\Gamma / \Delta$ is derivable from $\mathcal{R}$.

A Heyting algebra $A$ validates a multi-conclusion rule $\Gamma / \Delta$ provided for every valuation $v$ on $A$, if $v(\gamma)=1$ for all $\gamma \in \Gamma$, then $v(\delta)=1$ for some $\delta \in \Delta$. If $A$ validates $\Gamma / \Delta$, we write $A \models \Gamma / \Delta$. The following result is proved in $[4,18]$ :

TheOREM 2.2. $\Gamma / \Delta$ is derivable from $\mathcal{R}$ iff every Heyting algebra validating all rules in $\mathcal{R}$ validates also $\Gamma / \Delta$. 
We will say that rules $\rho_{1}$ and $\rho_{2}$ are equivalent if $\rho_{1}$ is derivable from $\left\{\rho_{2}\right\}$ and $\rho_{2}$ is derivable from $\left\{\rho_{1}\right\}$. By Theorem 2.2 this means that a Heyting algebra validates $\rho_{1}$ if and only if it validates $\rho_{2}$.

Derivability should be contrasted with admissibility; we will call a rule $\Gamma / \Delta$ admissible in IPC (or admissible tout court) iff it is valid in the free Heyting algebra with countably many generators. Taking into consideration the disjunction property of IPC, it is known (see e.g. [16,24]) that this is equivalent to either one of the following conditions: (1) every substitution making all members of $\Gamma$ a theorem in IPC makes also some member of $\Delta$ a theorem of IPC, and (2) adding $\Gamma / \Delta$ to IPC does not lead to the derivability of new theorems.

A set of rules $\mathcal{R}$ is said to form an admissible basis for a logic $L$ if every rule admissible in $L$ is derivable from $\mathcal{R}$.

\subsection{Closed Domain Condition and Stable Canonical Rules for Heyting Algebras}

We recall some definitions and results from [1].

Definition 2.3. Let $\mathfrak{X}=(X, \leq)$ and $\mathfrak{Y}=(Y, \leq)$ be Esakia spaces and let $f: X \rightarrow Y$ be a map. We call $f$ stable if it is continuous and orderpreserving.

It can be shown that Definition 2.3 can be dualized in the following way. Let $A$ and $B$ be Heyting algebras; then $h: A \rightarrow B$ is a bounded lattice morphism iff the dual Esakia morphism $h_{*}: B_{*} \rightarrow A_{*}$ is stable.

Definition 2.4. Let $\mathfrak{X}=(X, \leq)$ and $\mathfrak{Y}=(Y, \leq)$ be Esakia spaces, $f: X \rightarrow$ $Y$ be a map, and $U$ be a clopen subset of $Y$. We say that $f$ satisfies the closed domain condition (CDC) for $U$ if

$$
U \cap \uparrow f(x) \neq \varnothing \Rightarrow U \cap f(\uparrow x) \neq \varnothing
$$

holds for all $x \in X$. Let $\mathfrak{D}$ be a collection of clopen subsets of $Y$. We say that $f: X \rightarrow Y$ satisfies the closed domain condition (CDC) for $\mathfrak{D}$ if $f$ satisfies CDC for each $U \in \mathfrak{D}$.

Stable canonical rules are introduced in the following definition:

Definition 2.5. Let $A$ be a finite Heyting algebra and let $D \subseteq A^{2}$. For every $a \in A$ let $p_{a}$ be a propositional letter, and define the stable canonical rule $\rho(A, D)$ associated with $A$ and $D$ as $\gamma(A, D)=\Gamma / \Delta$, where 


$$
\begin{aligned}
\Gamma= & \left\{p_{0} \leftrightarrow 0\right\} \cup\left\{p_{1} \leftrightarrow 1\right\} \cup \\
& \left\{p_{a \vee b} \leftrightarrow\left(p_{a} \vee p_{b}\right) \mid a, b \in A\right\} \cup \\
& \left\{p_{a \wedge b} \leftrightarrow\left(p_{a} \wedge p_{b}\right) \mid a, b \in A\right\} \cup \\
& \left\{p_{a \rightarrow b} \leftrightarrow\left(p_{a} \rightarrow p_{b}\right) \mid(a, b) \in D\right\}
\end{aligned}
$$

and

$$
\Delta=\left\{p_{a} \leftrightarrow p_{b}: a, b \in A \text { with } a \neq b\right\} .
$$

Sometimes, if $F$ is the dual space of $A$, we might write $\gamma(F, D)$ instead of $\gamma(A, D)$.

Theorem 2.6. ([2, Proposition 3.2]) Let $A$ be a finite Heyting algebra, $D \subseteq$ $A^{2}$, and $B$ be an arbitrary Heyting algebra. Then the following are equivalent:

(i) $B \not \models \gamma(A, D)$;

(ii) there is a bounded lattice embedding $h: A \rightarrow B$ such that $h(a \rightarrow b)=$ $h(a) \rightarrow h(b)$ for each $(a, b) \in D$;

(iii) there is a stable onto map $f: B_{*} \rightarrow A_{*}$ satisfying CDC for $\mathfrak{D}:=$ $\{\alpha(a) \backslash \alpha(b):(a, b) \in D\}$.

The interesting point about stable rules is the following completeness theorem:

Theorem 2.7. ([2, Prop. 3.4]) Any intuitionistic multi-conclusion consequence relation can be axiomatized by stable canonical rules.

\section{Dichotomy Property and Admissible Basis for IPC}

Let $V_{n}$ be the rule:

$$
\frac{\left(\left(\bigvee_{i=1}^{n} p_{i}\right) \rightarrow q\right) \rightarrow \bigvee_{i=1}^{n} p_{i}}{q \rightarrow p_{1}|\cdots| q \rightarrow p_{n}}
$$

(denoted by $V_{n}^{\prime}$ in [17]).

THEOREM 3.1. The rule $V_{n}$ is admissible for each $n \in \omega$.

Proof. We have to show that if $\sigma$ is a substitution such that none of $\sigma q \rightarrow$ $\sigma p_{1}, \ldots, \sigma q \rightarrow \sigma p_{n}$ is a theorem of IPC, then $\left(\bigvee_{i=1}^{n} \sigma p_{i} \rightarrow \sigma q\right) \rightarrow \bigvee_{i=1}^{n} \sigma p_{i}$ is not a theorem either. By the finite model property of IPC there are finite rooted Kripke models $\mathfrak{M}_{1}, \ldots, \mathfrak{M}_{n}$ such that $\mathfrak{M}_{1} \models \sigma q, \ldots, \mathfrak{M}_{n} \models \sigma q$ and $\mathfrak{M}_{1} \not \forall \sigma p_{1}, \ldots, \mathfrak{M}_{n} \not \forall \sigma p_{n}$ (a Kripke model is said to be rooted iff its underlying poset has a root, i.e. a smallest element). Consider the disjoint 
union of $\mathfrak{M}_{1}, \ldots, \mathfrak{M}_{n}$ and add a new root $r$ to it. Extend the valuation to the resulting frame by making each variable false at $r$. Denote the new model by $\mathfrak{M}$. Then $\mathfrak{M}, r \not \neq \sigma p_{1}, \ldots, \sigma p_{n}$. So, $\mathfrak{M}, r \not \neq \bigvee_{i=1}^{n} \sigma p_{i}$ and $\mathfrak{M}, r \models \bigvee_{i=1}^{n} \sigma p_{i} \rightarrow$ $\sigma q$. Thus $\mathfrak{M}, r \forall\left(\bigvee_{i=1}^{n} \sigma p_{i} \rightarrow \sigma q\right) \rightarrow \bigvee_{i=1}^{n} \sigma p_{i}$. Hence $\left(\bigvee_{i=1}^{n} \sigma p_{i} \rightarrow \sigma q\right) \rightarrow$ $\bigvee_{i=1}^{n} \sigma p_{i}$ is not a theorem of IPC.

LEMMA 3.2. Suppose that a stable canonical rule $\gamma(F, D)$ has the following property. Given an Esakia space $W$ and a clopen up-set $Y \subseteq W$ and a stable surjective map $f: Y \rightarrow F$ satisfying $C D C$ for $D$, there is stable surjective $\bar{f}: W \rightarrow F$ with $f \subseteq \bar{f}$ satisfying $C D C$ for $D$. Then $\gamma(F, D)$ is equivalent to an assumption-free rule.

Proof. Let $\gamma(A, D)$ be the rule

$$
\frac{\varphi}{\varphi_{1}|\cdots| \varphi_{n}}
$$

We will show that under the assumption of the lemma this rule is equivalent to

$$
\overline{\varphi \rightarrow \varphi_{1}|\cdots| \varphi \rightarrow \varphi_{n}}
$$

$(2) \Rightarrow(1)$ is clear. Now assume that (the Heyting algebra dual to the Esakia space) $W$ does not validate (2). We show that then it does not validate (1). Let $V$ be a valuation on $W$ such that $V(\varphi) \nsubseteq V\left(\varphi_{1}\right), \ldots, V(\varphi) \nsubseteq V\left(\varphi_{n}\right)$. We set $Y=V(\varphi) \subseteq W$. Then $Y \not(1)$. This means that there is a stable surjective $f: Y \rightarrow F$. By the condition of the lemma $f$ is extended to stable surjective $\bar{f}: W \rightarrow F$, implying $W \not \models(1)$.

The following definition will be our main ingredient for a semantic characterization of admissibility of a stable canonical rule ${ }^{1}$ :

Definition 3.3. A stable canonical rule $\gamma(A, D)$ is called trivial if for all $S \subseteq A_{*}$ there is $x \in A_{*}$ such that

(1) $S \subseteq \uparrow x$

(2) For all $d \in D$ if $d \cap \uparrow x \neq \emptyset$, then $d \cap(\{x\} \cup \uparrow S) \neq \emptyset$.

We will see below that the triviality condition plays the same role for stable canonical rules as the existence of tight predecessors in the context of [18, Theorem 4.9 (iv)].

\footnotetext{
${ }^{1}$ The triviality notion below was independently introduced by J. Goudsmit in his thesis [14, Definition 4.76] under the name of 'adequate extendibility'. The author uses this notion when revisiting Rybakov results on admissible rules via universal (finite variable) models.
} 
THEOREM 3.4. The following are equivalent:

(1) $\gamma(A, D)$ is admissible.

(2) $\gamma(A, D)$ is derivable from $\left\{V_{n}: n \in \omega\right\}$.

(3) $\gamma(A, D)$ is not trivial.

(4) $\gamma(A, D)$ is not equivalent to an assumption-free rule.

Proof. $(2) \Rightarrow(1)$. We know that all $V_{n}$ are admissible, i.e. valid in the free Heyting algebra on infinitely many generators. Since moreover $\gamma(A, D)$ is derivable from $\left\{V_{n}: n \in \omega\right\}$, we conclude that $\gamma(A, D)$ is also valid on this algebra, i.e. is admissible.

$(3) \Rightarrow(2)$. Let $F=A_{*}$ and suppose $\gamma(F, D)$ is not derivable from $\left\{V_{n}: n \in \omega\right\}$. Then, by Theorem 2.2, there is an Esakia space $W$ validating all $V_{n}$ 's and refuting $\gamma(F, D)$. The latter means that there is a stable surjective $f: W \rightarrow F$ satisfying CDC for $D$. We will now show that $\gamma(F, D)$ is trivial. In what follows, we will employ the Heyting algebra $W^{*}$; in particular, implication will be understood in the sense of this algebra. Fix $S \subseteq F$. For $s \in S$ let $p_{s}=f^{-1}(F \backslash \downarrow s) \subseteq W, q=f^{-1}(\uparrow S)$. Since $f$ is stable, $p_{s}$ and $q$ are up-sets. For all $s \in S$ we have $q \nsubseteq p_{s}$. Indeed, if $x_{s} \in f^{-1}(s)$, then we have that $x_{s} \in q$ but $x_{s} \notin p_{s}$.

Since $W$ validates the rules $V_{n}$ for each $n \in \omega$, and none of $q \rightarrow p_{s}$ are the whole of $W$, it follows that neither $\left(\left(\bigcup_{s \in S} p_{s}\right) \rightarrow q\right) \rightarrow \bigcup_{s \in S} p_{s}$ is the whole of $W$; in particular, $\left(\left(\bigcup_{s \in S} p_{s}\right) \rightarrow q\right) \backslash\left(\bigcup_{s \in S} p_{s}\right)$ is not empty. As the topology on $F$ is discrete, $p_{s}$ and $q$ are clopen sets. Thus both $\left(\bigcup_{s \in S} p_{s}\right) \rightarrow q$ and $\bigcup_{s \in S} p_{s}$ are clopen too, and we may actually pick a maximal element $y$ of $\left(\left(\bigcup_{s \in S} p_{s}\right) \rightarrow q\right) \backslash \bigcup_{s \in S} p_{s}$.

We claim that then for each $y^{\prime}>y$ we have $y^{\prime} \in q$. Indeed since $\left(\bigcup_{s \in S} p_{s}\right) \rightarrow q$ is an upset and $y$ belongs to it, also $y^{\prime}$ will belong to it. But then $y^{\prime} \notin \bigcup_{s \in S} p_{s}$ is impossible by maximality of $y$, so $y^{\prime} \in \bigcup_{s \in S} p_{s}$, hence $y^{\prime} \in q$.

Let us now check that $f(y)$ fulfils the triviality conditions for $S$. For the first condition just note that $y \notin \bigcup_{s \in S} p_{s}$ iff for all $s \in S$ we have $y \notin f^{-1}(F \backslash \downarrow s)$, i.e. $y \in \bigcap_{s \in S} f^{-1}(\downarrow s)$, which is equivalent to $\uparrow f(y) \supseteq S$. For the second condition, suppose $d \cap \uparrow f(y) \neq \emptyset$ for $d \in D$, then by the CDC of $f$ we have that there is $y^{\prime} \geq y$ such that $f\left(y^{\prime}\right) \in d$. Thus, either $y^{\prime}=y$ and then $f\left(y^{\prime}\right)=f(y) \in d \cap\{f(y)\}$ or $y^{\prime}>y$ and then, as we have seen, $y^{\prime} \in q=f^{-1}(\uparrow S)$, so $f\left(y^{\prime}\right) \in d \cap \uparrow S$. Thus $\gamma(A, D)$ is trivial.

$(4) \Rightarrow(3)$ Suppose $\gamma(A, D)$ is trivial. We show that then it is equivalent to an assumption-free rule. We use Lemma 3.2. Let $W$ be an Esakia space, $Y \subseteq W$ a clopen up-set and $f: Y \rightarrow F$ a stable surjective map satisfying 
CDC for $D$. We extend $f$ to some $f^{l}: W \rightarrow F$ with the same properties. For $w \in W$ let $f_{w}=f(Y \cap \uparrow w)$. If $S \subseteq F$ is of the kind $f_{w}$, let $Y_{S} \subseteq W$ be $Y_{S}=\left\{w \in W \backslash Y: f_{w}=S\right\}$. We take a minimal $S \subseteq F$ such that $Y_{S} \neq \emptyset$ and extend $f$ to $Y \cup Y_{S}$.

Claim 3.5. $Y \cup Y_{S}$ is a clopen up-set.

Proof. It follows from the minimality of $S$ that $Y \cup Y_{S}$ is an up-set. Indeed, if $x \in Y \cup Y_{S}$ and $x \leqslant y$, then either $y \in Y$ and then we are done, or, provided $y \notin Y$, in view of minimality of $S, f_{y}=S$. Indeed since $Y$ is an up-set, $y \notin Y$ implies $x \notin Y$, so $x \in Y_{S}$, i.e. $f_{x}=S$. Moreover $\uparrow y \subseteq \uparrow x$, hence

$$
f_{y}=f(Y \cap \uparrow y) \subseteq f(Y \cap \uparrow x)=f_{x}=S,
$$

thus, as $S$ is minimal, $f_{y}=S$, i.e. $y \in Y_{S}$.

To show that $Y \cup Y_{S}$ is clopen it suffices to show that $Y_{S}$ is clopen. Now for any $w \in W$ we have that $w \in\left(W \backslash \downarrow f^{-1}(F \backslash S)\right) \backslash Y$ if and only if $f(Y \cap \uparrow w) \subseteq S$ and $w \notin Y$, which by minimality of $S$ is equivalent to $f(Y \cap \uparrow w)=S$ and $w \notin Y$. Thus $Y_{S}=\left(W \backslash \downarrow f^{-1}(F \backslash S)\right) \backslash Y$ is clopen. This finishes the proof of the claim.

We now extend $f$ to $\bar{f}$ with $\operatorname{dom}(\bar{f})=Y \cup Y_{S}$. We put

$$
\bar{f}(w)= \begin{cases}f(w), & \text { if } w \in Y, \\ s, & \text { if } w \notin Y,\end{cases}
$$

where $s$ is such that $S \subseteq \uparrow s$ and for all $d \in D, d \cap \uparrow s \neq \emptyset \Rightarrow d \cap(\{s\} \cup \uparrow S) \neq \emptyset$.

It is easy to see that $\bar{f}$ is order-preserving. Now we also show that $\bar{f}$ is continuous. Indeed, for every $x \in F$ we have $\bar{f}^{-1}(x)=f^{-1}(x)$ or $\bar{f}^{-1}(x)=$ $f^{-1}(x) \cup Y_{S}$. Since $Y_{S}$ is a clopen set the continuity follows.

Finally, we show that $\bar{f}$ satisfies CDC. The relevant case is when $d \cap$ $\uparrow \bar{f}(w) \neq \emptyset$ for $d \in D, w \in Y_{S}$. Now $\uparrow \bar{f}(w)=\uparrow s$. Thus, we have $d \cap(\{s\} \cup$ $\uparrow S) \neq \emptyset$ by the choice of $s$. Hence either $\bar{f}(w) \in d$ or there is $s^{\prime} \in S$ with $d \cap \uparrow s^{\prime} \neq \emptyset$. But $f_{w}=S$ (because $\left.w \in Y_{S}\right)$, hence $f(Y \cap \uparrow w)=S$. So there is $w^{\prime} \geq w$ such that $w^{\prime} \in Y$ and $f\left(w^{\prime}\right)=s^{\prime}$. We can then apply CDC for $f$ to get $w^{\prime \prime} \geq w^{\prime}$ with $f\left(w^{\prime \prime}\right) \in d$. Thus, $w^{\prime \prime} \geq w$ and $\bar{f}\left(w^{\prime \prime}\right)=f\left(w^{\prime \prime}\right) \in d$.

So we extended $f$ to $\bar{f}$ on $Y \cup Y_{S}$. We need to show that by repeating this procedure we cover the whole of $W$. This holds since the following is true: if some $S \subseteq F$ has been used for further extension of the map according to the above procedure, then this same $S$ can never occur again during any subsequent extensions. 
Indeed let $f^{k}$, resp. $f^{n}$ be any further extensions of $f$ to $Y^{k}$, resp. $Y^{n}$, $k<n<\omega$. Suppose we have used some $S$ for $f^{k}$; then it cannot happen that $S$ can be also used for $f^{n}$.

Suppose, to the contrary, that $S$ occurs as one of the candidates to build $f^{n}$. Then in particular $S=f_{w}^{n-1}$ for some $w \in Y^{n} \backslash Y^{n-1}$. Then also $w \notin Y^{k}$, so $S \neq f_{w}^{k-1}$ (since $Y^{k}=Y^{k-1} \cup Y_{S}^{k-1}$ and $Y_{S}^{k-1}$ consists precisely of those $v$ for which $f_{v}^{k-1}=S$ ). In fact by the minimality of $S, f_{w}^{k-1}$ cannot be included in $S$, so $f_{w}^{k-1} \backslash S$ is nonempty. Now note that since $f^{n-1}$ is an extension of $f^{k-1}$, one has $f_{w}^{n-1} \supseteq f_{w}^{k-1}$, hence also $f_{w}^{n-1} \backslash S$ is nonempty, which contradicts the equality $S=f_{w}^{n-1}$ above.

It thus follows that after each next extension at least one subset of $F$ is excluded from all subsequent extension steps. Thus after some step $n$ there will be no $w \notin Y^{n}$ and no $S$ left with the property $f_{w}^{n}=S$. Which just means that there is no $w$ outside $Y^{n}$, i.e. $Y^{n}=W$.

$(1) \Rightarrow(4)$ Suppose $\gamma(A, D)$ is admissible and equivalent to an assumptionfree rule $/ \Delta$. Then by the definition of admissibility any substitution makes one of the formulas in $\Delta$ a theorem of IPC. Hence $/ \Delta$ is valid on any Heyting algebra. However, $A \not \models \gamma(A, D)$, which is a contradiction.

Corollary 3.6. A stable canonical rule $\gamma(A, D)$ has the following dichotomy property: it is either admissible or equivalent to an assumption-free rule.

Corollary 3.7. Admissibility in IPC is decidable.

Proof. Given a rule $\rho$, we effectively compute the stable canonical rules $\gamma\left(A_{1}, D_{1}\right), \ldots, \gamma\left(A_{n}, D_{n}\right)$ which are equivalent to $\rho$ over IPC $[1,2]$. We will briefly sketch this algorithm. All the details can be found in [1, Thms 5.1, 5.5] and [2, Props 3.3, 3.4].

Let $\Xi$ be the set of all subformulas of formulas in $\Gamma \cup \Delta$. Then $\Xi$ is finite. Let $m$ be the cardinality of $\Xi$. Since the bounded lattice reduct of Heyting algebras is locally finite, up to isomorphism, there are only finitely many pairs $(A, D)$ satisfying the following two conditions:

(i) $A$ is a finite Heyting algebra that is at most $m$-generated as a bounded distributive lattice and $A \not \models \Gamma / \Delta$.

(ii) $D:=\{(v(\varphi), v(\psi)) \mid \varphi \rightarrow \psi \in \Xi\}$, where $v$ is a valuation on $A$ witnessing $A \not \models \Gamma / \Delta$.

Let $\left(A_{1}, D_{1}\right), \ldots,\left(A_{n}, D_{n}\right)$ be the enumeration of all such pairs and let $\gamma\left(A_{1}, D_{1}\right), \ldots, \gamma\left(A_{n}, D_{n}\right)$ be the corresponding stable canonical rules. Then $\gamma\left(A_{1}, D_{1}\right), \ldots, \gamma\left(A_{n}, D_{n}\right)$ are equivalent to $\rho$, i.e., for each Heyting algebra $B$ we have 


$$
B \models \rho \text { iff } B \models \gamma\left(A_{i}, D_{i}\right) \text { for each } i=1, \ldots, n \text {. }
$$

Hence $\rho$ is admissible if and only if each of the $\gamma\left(A_{1}, D_{1}\right), \ldots, \gamma\left(A_{n}, D_{n}\right)$ is admissible. By Theorem 3.4, each $\gamma\left(A_{i}, D_{i}\right)$ is admissible iff it is not trivial. Obviously, triviality of a rule $\gamma\left(A_{i}, D_{i}\right)$ can be checked in finite time. The result follows. ${ }^{2}$

COROllary 3.8. The rules $\left\{V_{n}: n \in \omega\right\}$ form an admissible basis for IPC. Proof. By Theorem 2.7 and the above.

\section{Preliminaries on Modal Algebras and Modal Logics}

\subsection{Duality for Modal Algebras}

We use $[5,6,19,28]$ as our main references for the basic theory of normal modal logics, including their algebraic and relational semantics, and the dual equivalence between modal algebras and modal spaces (descriptive Kripke frames).

A modal algebra is a pair $\mathfrak{A}=(A, \diamond)$, where $A$ is a Boolean algebra and $\diamond$ is a unary operator on $A$ that commutes with finite joins. As usual, the dual operator $\square$ is defined as $\neg \diamond \neg$. A modal homomorphism between two modal algebras is a Boolean homomorphism $h$ satisfying $h(\diamond a)=\diamond h(a)$. Let MA be the category of modal algebras and modal homomorphisms.

A modal space (or descriptive Kripke frame) is a pair $\mathfrak{X}=(X, R)$, where $X$ is a Stone space (zero-dimensional compact Hausdorff space) and $R$ is a binary relation on $X$ satisfying the conditions:

$$
R[x]:=\{y \in X: x R y\}
$$

is closed for each $x \in X$ and

$$
R^{-1}[U]:=\{x \in X: \exists y \in U \text { with } x R y\}
$$

is clopen (closed and open) for each clopen $U$ of $X$. A bounded morphism (or $p$-morphism) $f: \mathfrak{X} \rightarrow \mathfrak{Y}$ between two modal spaces is a continuous map $f: X \rightarrow Y$ such that $f(R[x])=R[f(x)]$ for all $x \in X$. Let MS be the category of modal spaces and bounded morphisms.

It is a well-known theorem in modal logic that MA is dually equivalent to MS. The functors $(-)_{*}:$ MA $\rightarrow$ MS and $(-)^{*}:$ MS $\rightarrow$ MA that establish this dual equivalence are constructed as follows. For a modal algebra $\mathfrak{A}=$

\footnotetext{
${ }^{2}$ An alternative proof can be given as follows: rule admissibility is $\Pi_{1}^{0}$ and derivability from a recursive set of rules $\left\{V_{n}: n \in \omega\right\}$ is $\Sigma_{1}^{0}$. Thus, admissibility is decidable.
} 
$(A, \diamond)$, let $\mathfrak{A}_{*}=\left(A_{*}, R\right)$, where $A_{*}$ is the Stone space of $A$ (that is, the set of ultrafilters of $A$ topologized by the basis $\{\beta(a): a \in A\}$, where $\left.\beta(a)=\left\{x \in A_{*}: a \in x\right\}\right)$ and $x R y$ iff $(\forall a \in A)(a \in y \Rightarrow \nabla a \in x)$. We call $R$ the dual of $\diamond$. For a modal homomorphism $h$, let $h_{*}=h^{-1}$. For a modal space $\mathfrak{X}=(X, R)$, let $\mathfrak{X}^{*}=(A, \diamond)$, where $A$ is the Boolean algebra of clopens of $X$ and $\diamond(U)=R^{-1}[U]$. For a bounded morphism $f$, let $f^{*}=f^{-1}$.

Let $\mathfrak{A}=(A, \diamond)$ be a modal algebra and let $\mathfrak{X}=(X, R)$ be its dual space. Then it is well known that $R$ is reflexive iff $a \leqslant \diamond a$ for all $a \in A$, and $R$ is transitive iff $\nabla \nabla a \leqslant \diamond a$ for all $a \in A$. A modal algebra $\mathfrak{A}$ is a K4-algebra if $\diamond \diamond a \leqslant \diamond a$ holds in $\mathfrak{A}$, and it is an S4-algebra if in addition $a \leqslant \triangleright a$ holds in $\mathfrak{A}$. S4-algebras are also known as closure algebras, interior algebras, or topological Boolean algebras. Let K4 be the full subcategory of MA consisting of $\mathbf{K} 4$-algebras, and let S4 be the full subcategory of K4 consisting of $\mathbf{S} 4$-algebras. A modal space $\mathfrak{X}=(X, R)$ is a transitive space if $R$ is transitive, and it is a quasi-ordered space if $R$ is reflexive and transitive.

For a clopen subset $Y \subseteq X$ of a transitive space $(X, R)$, a point $y \in Y$ is called quasi-maximal if for any $x \in Y$ with $y R x$ we have $x R y$. It is known that any point of any clopen subset sees a quasi-maximal point of this subset (see e.g. [6, Theorem 10.36]).

Let TS be the full subcategory of MS consisting of transitive spaces, and let QS be the full subcategory of TS consisting of quasi-ordered spaces. Then the dual equivalence of MA and MS restricts to the dual equivalence of K4 and TS, which restricts further to the dual equivalence of S4 and QS.

\subsection{Multi-conclusion Modal Rules}

We use greek letters $\gamma, \delta, \ldots, \varphi, \psi, \ldots$ to denote formulas built up from propositional variables using the connectives $\neg, \wedge, \vee, \rightarrow, \perp, \top, \diamond$. A valuation on a modal algebra $\mathfrak{A}=(A, \diamond)$ is a map associating an element of $A$ with every propositional variable. It is then extended to all modal formulas in a standard way. A Kripke frame is a pair $(X, R)$ where $X$ is a set and $R$ is a binary relation on $X$. A Kripke model is a triple $(X, R, V)$, where $(X, R)$ is a Kripke frame and $V$ is a valuation on the powerset Boolean algebra of $X$ with $\diamond:=R^{-1}$. We use letters $\mathfrak{M}, \mathfrak{N}, \ldots$ for Kripke models and the notation $\mathfrak{M}, x \models \varphi$ to mean that $x$ belongs to $V(\varphi)$, where $V$ is the valuation of the Kripke model $\mathfrak{M}$. The notation $\mathfrak{M} \models \varphi$ (' $\varphi$ is valid in $\mathfrak{M}$ ') means that $\mathfrak{M}, x \models \varphi$ holds for all $x$ from the underlying frame of $\mathfrak{M}$. We let $\mathbf{K}$, K4, S4 stand for the set of formulas which are valid in all modal algebras, K4-modal algebras, S4-modal algebras, respectively (as it is well-known, 
we can equivalently use validity in the corresponding classes of Kripke models).

A transitive normal modal multi-conclusion consequence relation is a set $\mathcal{S}$ of modal rules such that

(1) $\varphi / \varphi \in \mathcal{S}$.

(2) $\varphi, \varphi \rightarrow \psi / \psi \in \mathcal{S}$.

(3) $\varphi / \square \varphi \in \mathcal{S}$.

(4) $/ \varphi \in \mathcal{S}$ for each $\varphi$ in $\mathbf{K} 4$.

(5) If $\Gamma / \Delta \in \mathcal{S}$, then $\Gamma, \Gamma^{\prime} / \Delta, \Delta^{\prime} \in \mathcal{S}$.

(6) If $\Gamma / \Delta, \varphi \in \mathcal{S}$ and $\Gamma, \varphi / \Delta \in \mathcal{S}$, then $\Gamma / \Delta \in \mathcal{S}$.

(7) If $\Gamma / \Delta \in \mathcal{S}$ and $\sigma$ is a substitution, then $\sigma(\Gamma) / \sigma(\Delta) \in \mathcal{S}$.

We denote the least transitive normal modal multi-conclusion consequence relation by $\mathbf{S}_{\mathbf{K} 4}$. For a set $\mathcal{R}$ of multi-conclusion modal rules, let $\mathbf{S}_{\mathbf{K} 4}+\mathcal{R}$ be the least transitive normal modal multi-conclusion consequence relation containing $\mathcal{R}$. If $\mathcal{S}=\mathbf{S}_{\mathbf{K} 4}+\mathcal{R}$, then we say that $\mathcal{S}$ is axiomatized by $\mathcal{R}$ or that $\mathcal{R}$ is a basis for $\mathcal{S}$. Whenever $\Gamma / \Delta$ belongs to $\mathbf{S}_{\mathbf{K} 4}+\mathcal{R}$ we say that $\Gamma / \Delta$ is derivable from $\mathcal{R}$.

A K4 algebra $A$ validates a multi-conclusion rule $\Gamma / \Delta$ provided for every valuation $v$ on $A$, if $v(\gamma)=1$ for all $\gamma \in \Gamma$, then $v(\delta)=1$ for some $\delta \in \Delta$. If $A$ validates $\Gamma / \Delta$, we write $A=\Gamma / \Delta$. The following result is proved in $[4,18]$ :

THEOREM 4.1. $\Gamma / \Delta$ is derivable from $\mathcal{R}$ iff every $\mathbf{K} 4$-algebra validating all rules in $\mathcal{R}$ also validates $\Gamma / \Delta$.

Admissibility of rules in modal calculi is defined similarly to the intuitionistic case (described in 2.2) and has similar properties.

\subsection{Closed Domain Conditions and Stable Canonical Rules for Modal Algebras}

We now introduce the key concepts of stable homomorphisms and the closed domain condition, and show how the two relate to each other. For the proofs of the results stated in this subsection, the reader is referred to [1].

Definition 4.2. Let $\mathfrak{A}=(A, \diamond)$ and $\mathfrak{B}=(B, \diamond)$ be $\mathbf{K} 4$-algebras and let $h: A \rightarrow B$ be a Boolean homomorphism. We call $h$ a stable homomorphism provided $\diamond h(a) \leqslant h(\diamond a)$ for each $a \in A$. 
It is easy to see that $h: A \rightarrow B$ is stable iff $h(\square a) \leq \square h(a)$ for each $a \in A$. Stable homomorphisms were considered in [3] under the name of semi-homomorphisms and in [13] under the name of continuous morphisms.

Definition 4.3. Let $\mathfrak{X}=(X, R)$ and $\mathfrak{Y}=(Y, R)$ be transitive modal spaces and let $f: X \rightarrow Y$ be a map. We call $f$ stable if it is continuous and $x R y$ implies $f(x) R f(y)$.

Lemma 4.4. Let $\mathfrak{A}=(A, \diamond)$ and $\mathfrak{B}=(B, \diamond)$ be K4-algebras, $\mathfrak{X}=(X, R)$ be the dual of $\mathfrak{A}, \mathfrak{Y}=(Y, R)$ be the dual of $\mathfrak{B}$, and $h: A \rightarrow B$ be a Boolean homomorphism. Then $h: A \rightarrow B$ is stable iff $h_{*}: Y \rightarrow X$ is stable.

Definition 4.5. Let $\mathfrak{X}=(X, R)$ and $\mathfrak{Y}=(Y, R)$ be transitive modal spaces, $f: X \rightarrow Y$ be a map, and $U$ be a clopen subset of $Y$. We say that $f$ satisfies the closed domain condition (CDC) for $U$ if

$$
R[f(x)] \cap U \neq \varnothing \Rightarrow f(R[x]) \cap U \neq \varnothing .
$$

Let $\mathfrak{D}$ be a collection of clopen subsets of $Y$. We say that $f: X \rightarrow Y$ satisfies the closed domain condition (CDC) for $\mathfrak{D}$ if $f$ satisfies CDC for each $U \in \mathfrak{D}$.

Theorem 4.6. Let $\mathfrak{A}=(A, \diamond)$ and $\mathfrak{B}=(B, \diamond)$ be K4-algebras, $h: A \rightarrow B$ be a stable homomorphism, and $a \in A$. The following two conditions are equivalent:

(1) $h(\diamond a)=\diamond h(a)$.

(2) $h_{*}: B_{*} \rightarrow A_{*}$ satisfies CDC for $\beta(a)$.

Theorem 4.6 motivates the following definition.

Definition 4.7. Let $\mathfrak{A}=(A, \diamond)$ and $\mathfrak{B}=(B, \diamond)$ be $\mathbf{K} 4$-algebras and let $h: A \rightarrow B$ be a stable homomorphism.

(1) We say that $h$ satisfies the closed domain condition (CDC) for $a \in A$ if $h(\diamond a)=\diamond h(a)$.

(2) We say that $h$ satisfies the closed domain condition (CDC) for $D \subseteq A$ if $h$ satisfies CDC for each $a \in D$.

We now come to stable canonical rules:

Definition 4.8. Let $\mathfrak{A}=(A, \diamond)$ be a finite $\mathbf{K} 4$-algebra and let $D$ be a subset of $A$. For each $a \in A$ we introduce a new propositional letter $p_{a}$ and define the stable canonical rule $\rho(\mathfrak{A}, D)$ associated with $\mathfrak{A}$ and $D$ as $\Gamma / \Delta$, where: 


$$
\begin{aligned}
\Gamma= & \left\{p_{a \vee b} \leftrightarrow p_{a} \vee p_{b}: a, b \in A\right\} \cup \\
& \left\{p_{\neg a} \leftrightarrow \neg p_{a}: a \in A\right\} \cup \\
& \left\{p_{a} \rightarrow p_{\diamond a}: a \in A\right\} \cup \\
& \left\{p_{\diamond a} \rightarrow \diamond p_{a}: a \in D\right\},
\end{aligned}
$$

and

$$
\Delta=\left\{p_{a}: a \in A, a \neq 1\right\} .
$$

Stable canonical rules are characterized in terms of refutations as follows: Theorem 4.9. Let $\mathfrak{A}=(A, \diamond)$ be a finite K4-algebra, $D \subseteq A$, and $\mathfrak{B}=$ $(B, \diamond)$ be a K4-algebra. Then $\mathfrak{B} \not \rho(\mathfrak{A}, D)$ iff there is a stable embedding $h: A \longmapsto B$ satisfying CDC for $D$.

It was proved in [1] that every multi-conclusion consequence relation above $\mathbf{K}$ is axiomatizable by stable canonical rules (relative to arbitrary finite modal algebras - not only to those validating $\mathbf{K} \mathbf{4}$-axiom). The same proof can easily be extended to our multi-conclusion consequence relations above K4. Thus, we have the following theorem.

THEOREM 4.10. Any transitive normal modal multi-conclusion consequence relation can be axiomatized by canonical rules $\rho(\mathfrak{A}, D)$ (where $\mathfrak{A}=(A, \diamond)$ is a finite $\mathbf{K 4}$-algebra and $D \subseteq A$ ).

\section{Dichotomy Property and Admissible Basis for K4}

From now on, all Kripke frames and modal spaces are assumed to be transitive. Below $\square^{+} \varphi$ abbreviates $\varphi \wedge \square \varphi$; in a transitive Kripke frame/modal space $(X, R), R^{+}$abbreviates $R \cup i d$ and $\uparrow S$ stands for $\{w \in X \mid \exists s \in$ $\left.S s R^{+} w\right\}$. We may also use the notation $\uparrow S$ for $\{w \in X \mid \exists s \in S s R w\}$. When we say that $S$ is an up-set we mean $S=\uparrow S$. If $S$ is a singleton set $\{y\}$, then we use $\uparrow y$ and $\uparrow y$ instead of $\uparrow\{y\}$ and $\uparrow\{y\}$, respectively. Notations $\downarrow S, \downarrow S, \downarrow\{y\}$ and $\downarrow\{y\}$ are defined dually (notice that $R^{-1}(S)$ is the same as $\downarrow S)$.

Let $F=(W, R)$ be a frame dual to a finite $\mathbf{K} 4$-algebra $\mathfrak{A}=(A, \diamond)$. We denote the set $\{\beta(a): a \in D\}$ by $D$. We will also denote (abusing notation) the stable canonical rule $\rho(\mathfrak{A}, D)$ by $\rho(F, D)$.

Let $S_{n}^{\ell, m}$ be the rule:

$$
\frac{\left(\bigwedge_{l=1}^{\ell}\left(\square v_{l} \rightarrow v_{l}\right) \wedge \bigwedge_{k=1}^{m} \square\left(r_{k} \rightarrow \square\left(r_{k} \vee \square^{+} q\right)\right)\right) \rightarrow \bigvee_{i=1}^{n} \square p_{i}}{\square^{+} q \rightarrow p_{1}|\cdots| \square^{+} q \rightarrow p_{n}}
$$


and $T_{n}^{m}$ be the rule:

$$
\frac{\bigwedge_{k=1}^{m}\left(\diamond r_{k} \rightarrow \diamond\left(r_{k} \wedge \square^{+} q\right)\right) \rightarrow \bigvee_{i=1}^{n} \square p_{i}}{\square^{+} q \rightarrow p_{1}|\cdots| \square^{+} q \rightarrow p_{n}}
$$

THEOREM 5.1.

(1) The rule $\left(S_{n}^{\ell, m}\right)$ is admissible in $\mathbf{K} \mathbf{4}$ for each $n, m, \ell \in \omega$.

(2) The rule $\left(T_{n}^{m}\right)$ is admissible in $\mathbf{K} \mathbf{4}$ for all $n, m \in \omega$.

Proof. The foregoing proof is essentially an adjustment of the proof of Theorem 3.1.

(1) We have to show that if $\sigma$ is a substitution such that none of $\square^{+} \sigma q \rightarrow \sigma p_{1}, \ldots, \square^{+} \sigma q \rightarrow \sigma p_{n}$ is a theorem of $\mathbf{K} 4$, then $\left(\bigwedge_{l=1}^{\ell}\left(\square \sigma v_{l} \rightarrow \sigma v_{l}\right) \wedge \bigwedge_{k=1}^{m} \square\left(\sigma r_{k} \rightarrow \square\left(\sigma r_{k} \vee \square^{+} \sigma q\right)\right)\right) \rightarrow \bigvee_{i=1}^{n} \square \sigma p_{i}$ is not a theorem either.

By the finite model property of $\mathbf{K} \mathbf{4}$ there are finite rooted Kripke models $\mathfrak{M}_{1}, \ldots, \mathfrak{M}_{n}$ such that $\mathfrak{M}_{i} \models \square^{+} \sigma q$ and $\mathfrak{M}_{i} \not \models \sigma p_{i}$ for all $i=1, \ldots, n$. Consider the disjoint union of $\mathfrak{M}_{1}, \ldots, \mathfrak{M}_{n}$ and add a new reflexive root $\varrho$ to it. Extend the valuation to the resulting frame by making each variable false at $\varrho$. Denote the new model by $\mathfrak{M}$. Then by reflexivity of $\varrho$ we will have $\mathfrak{M}, \varrho \models \bigwedge_{l=1}^{\ell}\left(\square \sigma v_{l} \rightarrow \sigma v_{l}\right)$. Moreover $\mathfrak{M}, \varrho \not \models \bigvee_{i=1}^{n} \square \sigma p_{i}$, because for every $i, \varrho$ sees a point in $\mathfrak{M}_{i}$ where $\sigma p_{i}$ is not true. Thus we will be done if $\mathfrak{M}, \varrho \models \bigwedge_{k=1}^{m} \square\left(\sigma r_{k} \rightarrow \square\left(\sigma r_{k} \vee \square^{+} \sigma q\right)\right)$. This means that for any $k$ and any $w$ in $\mathfrak{M}$ with $\mathfrak{M}, w \models \sigma r_{k}$ one has $\mathfrak{M}, w^{\prime}=\sigma r_{k} \vee \square^{+} \sigma q$ for all $w^{\prime}$ with $w R w^{\prime}$. But any such $w^{\prime}$ is either in some $\mathfrak{M}_{i}$ and then $\mathfrak{M}, w^{\prime} \models \square^{+} \sigma q$, or $w^{\prime}=\varrho$, and then because of $w R w^{\prime}$ also $w=\varrho$, so $\mathfrak{M}, w^{\prime} \models \sigma r_{k}$. In both cases $\mathfrak{M}, w^{\prime} \models \sigma r_{k} \vee \square^{+} \sigma q$.

(2) The rule $\left(T_{n}^{m}\right)$ is proved to be admissible in a similar way (this time, an irreflexive extra root is needed).

Lemma 5.2. Suppose that a stable canonical rule $\rho(F, D)$ has the following property. Given a transitive modal space $(W, R)$ and a clopen up-set $Y \subseteq W$ and a stable surjective map $f: Y \rightarrow F$ satisfying $C D C$ for $D$, there is stable surjective $\bar{f}: W \rightarrow F$ with $f \subseteq \bar{f}$ satisfying $C D C$ for $D$. Then $\rho(F, D)$ is equivalent to an assumption-free rule.

Proof. Let $\rho(A, D)$ be the rule

$$
\frac{\varphi}{\varphi_{1}|\cdots| \varphi_{n}}
$$


We will show that under the assumption of the lemma this rule is equivalent to

$$
\overline{\square^{+} \varphi \rightarrow \varphi_{1}|\cdots| \square^{+} \varphi \rightarrow \varphi_{n}}
$$

$(2) \Rightarrow(1)$ is clear. Now assume that a transitive modal space $(W, R)$ does not validate (2). We show that then it does not validate (1). Let $V$ be a valuation on $W$ such that $V\left(\square^{+} \varphi\right) \nsubseteq V\left(\varphi_{1}\right), \ldots, V\left(\square^{+} \varphi\right) \nsubseteq V\left(\varphi_{n}\right)$. We set $Y=V\left(\square^{+} \varphi\right) \subseteq W$. Then $Y \not \models(1)$. This means that there is a stable surjective $f: Y \rightarrow F$ satisfying CDC for $D$. By the condition of the lemma $f$ can be extended to a stable surjective map $\bar{f}: W \rightarrow F$ satisfying CDC for $D$, implying $W \not \models(1)$.

The following is a modal analogue of Definition 3.3.

Definition 5.3. A stable canonical rule $\rho(A, D)$ is called trivial ${ }^{\circ}$ if for all $S \subseteq A_{*}$ there is a reflexive $x^{\circ} \in A_{*}$ such that

(1) $S \subseteq \uparrow x^{\circ}$

(2) For all $d \in D$, if $d \cap \uparrow x^{\circ} \neq \emptyset$ then $d \cap\left(\left\{x^{\circ}\right\} \cup \uparrow S\right) \neq \emptyset$.

A stable canonical rule $\rho(A, D)$ is called trivial ${ }^{\bullet}$ if for all $S \subseteq A_{*}$ there is $x^{\bullet} \in A_{*}$ such that

(3) $S \subseteq \uparrow x^{\bullet}$

(4) For all $d \in D$, if $d \cap \uparrow x^{\bullet} \neq \emptyset$ then $d \cap \uparrow S \neq \emptyset$.

A stable canonical rule $\rho(A, D)$ is called trivial iff it is both trivial ${ }^{\circ}$ and trivial ${ }^{\bullet}{ }^{3}$

THEOREM 5.4. The following are equivalent:

(1) $\rho(A, D)$ is admissible.

(2) $\rho(A, D)$ is derivable from $\left\{S_{n}^{\ell, m}: m, n, \ell \in \omega\right\} \cup\left\{T_{n}^{m}: m, n \in \omega\right\}$.

(3) $\rho(A, D)$ is not trivial.

(4) $\rho(A, D)$ is not equivalent to an assumption-free rule.

Proof. (2) $\Rightarrow(1)$. We know that all $S_{n}^{\ell, m}$ and $T_{n}^{m}$ are admissible, i.e. valid in the free K4-algebra on infinitely many generators. Since moreover $\rho(A, D)$ is derivable from $\left\{S_{n}^{\ell, m}, T_{n}^{m}: \ell, m, n \in \omega\right\}$, we conclude that $\rho(A, D)$ is also valid on this algebra, i.e. is admissible.

\footnotetext{
${ }^{3}$ Notice that the points $x^{\circ}$ and $x^{\bullet}$ may coincide.
} 
$(3) \Rightarrow(2)$. Suppose $\rho(F, D)$ is not derivable from $\left\{S_{n}^{\ell, m}: m, n, \ell \in \omega\right\} \cup$ $\left\{T_{n}^{m}: m, n \in \omega\right\}$ with $F=A_{*}$. Then there is a transitive modal space $(W, R)$ validating all $S_{n}^{\ell, m}$ 's and all $T_{n}^{m}$ 's and refuting $\rho(F, D)$. The latter means that there is a stable surjective $f: W \rightarrow F$ satisfying $C D C$ for $D$. Fix $S \subseteq F$.

We will first show that there exist $x^{\circ}$ and $x^{\bullet}$ satisfying the conditions of Definition 5.3(1)-(2). In what follows we are working in the modal algebra $(W, R)^{*}$; all connectives and modal operators are taken in this algebra. For $s \in S$ let $p_{s}=W \backslash f^{-1}(s) \subseteq W$, let $q=f^{-1}(\uparrow S)$ and let $r_{k}=f^{-1}(k)$ for $k \in$ $F$. Let $C=\left\{v_{1}, \ldots, v_{\ell}\right\}$ be a finite set of clopens of $W$. Since $f$ is continuous and $F$ is discrete, $p_{s}$ and $r_{k}$ are clopens, while $q$ is a clopen up-set in $W$ since $f$ is also stable. In particular, $q$ and $\square^{+} q$ have the same underlying set. Moreover, for all $s \in S$ we have $\square^{+} q \nsubseteq p_{s}$. Indeed, for any $w_{s} \in f^{-1}(s)$ we have that $w_{s} \in q$ but $w_{s} \notin p_{s}=W \backslash f^{-1}(s)$. This means that the conclusion of the rule $S_{n}^{\ell, m}$ is falsified on $W$. It follows that $W$ falsifies the premise of that rule as well. Hence there exists $w_{C} \in W$ such that $w_{C} \in \bigcap_{l=1}^{\ell}\left(\square v_{l} \rightarrow\right.$ $\left.v_{l}\right), w_{C} \in \bigcap_{k \in F} \square\left(r_{k} \rightarrow \square\left(r_{k} \cup \square^{+} q\right)\right)$ and $w_{C} \notin \bigcup_{s \in S} \square p_{s}$. The latter can be equivalently written as $w_{C} \in \bigcap_{s \in S} \diamond f^{-1}(s)$. We thus obtain that the set $\left\{\square v \rightarrow v \mid v \in W^{*}\right\} \cup\left\{\bigcap_{k \in F} \square\left(r_{k} \rightarrow \square\left(r_{k} \cup \square^{+} q\right)\right) \cap \bigcap_{s \in S} \diamond f^{-1}(s)\right\}$ of clopens of $W$ has finite intersection property. Since $W$ is compact, the intersection of all these clopens is nonempty, i.e. there is $w \in W$ that belongs to all of these clopens. That is, $w$ belongs to all clopens of the form $\square v \rightarrow v$ (which means that $w$ is reflexive), and also $w \in \bigcap_{k \in F} \square\left(r_{k} \rightarrow \square\left(r_{k} \cup \square^{+} q\right)\right.$ ) and $w \in \bigcap_{s \in S} \diamond f^{-1}(s)$. By the latter, we have that for every $s \in S$ there is a $w^{\prime}$ such that $w R w^{\prime}$ and $f\left(w^{\prime}\right)=s$. In other words, $f$ being stable, $\uparrow f(w) \supseteq S$. Let $x:=f(w)$. Then condition (1) of Definition 5.3 is met (notice that $x$ is reflexive because $w$ is reflexive and $f$ is stable). We now show that condition (2) is met as well.

Since $w \in \bigcap_{k \in F} \square\left(r_{k} \rightarrow \square\left(r_{k} \cup \square^{+} q\right)\right)$, in particular we have $w \in \square\left(r_{x} \rightarrow\right.$ $\left.\square\left(r_{x} \cup \square^{+} q\right)\right)$. Since $w \in r_{x}$, we obtain that $w \in \square\left(r_{x} \cup \square^{+} q\right)=\square\left(\neg r_{x} \rightarrow\right.$ $\left.\square^{+} q\right)$. This means that any $w^{\prime}$ such that $w R w^{\prime}$ and $f\left(w^{\prime}\right) \neq x$ will be necessarily in $\square^{+} q$.

Now if $d \cap \uparrow x \neq \emptyset$ for some $d \in D$, then as $x=f(w)$, by the CDC of $f$ there is $w^{\prime}$ such that $w R w^{\prime}$ and $f\left(w^{\prime}\right) \in d$. Then, either $f\left(w^{\prime}\right)=x$ and then $f\left(w^{\prime}\right) \in d \cap\{x\}$, or $f\left(w^{\prime}\right) \neq x$ and then as we have seen $f\left(w^{\prime}\right) \in \uparrow S$. Thus $f\left(w^{\prime}\right) \in d \cap(\{x\} \cup \uparrow S)$, so that $d \cap(\{x\} \cup \uparrow S) \neq \emptyset$. This implies that $\rho(F, D)$ is trivial ${ }^{\circ}$ (putting $x^{\circ}=x$ ).

Next we show that there exists an $x^{\bullet}$ satisfying the conditions of Definition 5.3(3)-(4). As above, for $s \in S$ let $p_{s}=W \backslash f^{-1}(s) \subseteq W$, let $q=f^{-1}(\uparrow S)$ 
and let $r_{k}=f^{-1}(k)$ for $k \in F$. Again, the conclusion of the rule $T_{n}^{m}$ is falsified on $W$ and consequently $W$ falsifies the premise of that rule as well. Thus there is $w \in \bigcap_{k \in F}\left(\diamond r_{k} \rightarrow \diamond\left(r_{k} \wedge \square^{+} q\right)\right)$ and $w \notin \bigcup_{s \in S} \square p_{s}$. By the latter, we have that for every $s \in S$ there is a $w^{\prime}$ such that $w R w^{\prime}$ and $f\left(w^{\prime}\right)=s$. In other words, $f$ being stable, $\uparrow f(w) \supseteq S$. Let $x:=f(w)$. Then condition (3) of Definition 5.3 is met. For condition (4), consider $d \in D$ such that $d \cap \uparrow f(w) \neq \emptyset$. Then, since $f$ satisfies CDC for $D$, there is an $u \in W$ with $w R u$ and $f(u) \in d$. Thus $w \in \nabla r_{k}$ for $k=f(u)$, as $r_{k}=f^{-1}(k)$; since $w \in \diamond r_{k} \rightarrow \diamond\left(r_{k} \wedge \square^{+} q\right)$, there is $w^{\prime}$ such that $w R w^{\prime}$ and $w^{\prime} \in r_{k} \cap \square^{+} q$, which means in particular that $f\left(w^{\prime}\right)=k \in d \cap \uparrow S$, as wanted. Putting $x^{\bullet}=x$ we deduce that $\rho(F, D)$ is trivial $\bullet^{\bullet}$ and hence, trivial.

$(4) \Rightarrow(3)$. Suppose $\rho(A, D)$ is trivial. We show that then it is equivalent to an assumption-free rule. Using Lemma 5.2, it suffices to extend any stable surjective map $f: Y \rightarrow F$ from a clopen up-set $Y \subseteq W$ of a transitive modal space $(W, R)$ to $F$ satisfying CDC for $D$ to an $\bar{f}: W \rightarrow F$ with the same properties.

For $w \in W$ let $f_{w}=f(Y \cap \uparrow w)$. If $S \subseteq F$ is of the kind $f_{w}$, let $Y_{S} \subseteq W$ be $Y_{S}=\left\{w \in W \backslash Y: f_{w}=S\right\}$. We take a minimal $S \subseteq F$ such that $Y_{S} \neq \emptyset$ (i.e. that $S=f_{w}$ for some $w \notin Y$ ) and extend $f$ to $Y \cup Y_{S}$.

Claim 5.5. $Y \cup Y_{S}$ is a clopen up-set.

Proof. That $Y \cup Y_{S}$ is an up-set follows from minimality of $S$. Indeed, if $x \in Y \cup Y_{S}$ and $x R y$, then either $y \in Y$ and then we are done, or, provided $y \notin Y$, then, since $Y$ is an up-set, also $x \notin Y$, so $x \in Y_{S}$, i.e. $f_{x}=S$. Moreover $\uparrow y \subseteq \uparrow x$, hence

$$
f_{y}=f(Y \cap \uparrow y) \subseteq f(Y \cap \uparrow x)=f_{x}=S,
$$

so by minimality of $S$ necessarily $f_{y}=S$. The latter means $y \in Y_{S}$, so $y \in Y \cup Y_{S}$. Thus $Y \cup Y_{S}$ is an up-set.

To show that $Y \cup Y_{S}$ is clopen it suffices to show that $Y_{S}$ is clopen. Indeed, for any $w \in W$ we have that $w \in\left(\square^{+}\left(Y \rightarrow f^{-1} S\right)\right) \backslash Y$ if and only if $f(Y \cap \uparrow w) \subseteq S$ and $w \notin Y$, which by minimality of $S$ implies that actually $f(Y \cap \uparrow w)=S$. Thus $Y_{S}=\left(\square^{+}\left(Y \rightarrow f^{-1} S\right)\right) \backslash Y$ is clopen. This finishes the proof of the claim.

We now extend $f$ to $\bar{f}$ with $Y \varsubsetneqq \operatorname{dom} \bar{f}=Y \cup Y_{S}$. Recall that, by the triviality of $(F, D)$, there exist two (not necessarily distinct) points $s^{\bullet}, s^{\circ}$ such that (i) $S \subseteq \uparrow s^{\bullet}$ and $d \cap \uparrow s^{\bullet} \neq \emptyset \Rightarrow d \cap \uparrow S \neq \emptyset$ for all $d \in D$; (ii) $s^{\circ}$ is reflexive, $S \subseteq \uparrow s^{\circ}$ and $d \cap \uparrow s^{\circ} \neq \emptyset \Rightarrow d \cap\left(\left\{s^{\circ}\right\} \cup \uparrow S\right) \neq \emptyset$ for all $d \in D$. We distinguish two cases, depending whether $S$ has a reflexive root or not. 
Case (I): $S$ has a reflexive root $s \in S$. We put:

$$
\bar{f}(w)= \begin{cases}f(w), & \text { if } w \in Y, \\ s, & \text { if } w \in Y_{S} \backslash Y .\end{cases}
$$

It is easy to see that $\bar{f}$ is stable ( $s$ is reflexive). Now we also show that $\bar{f}$ is continuous. Indeed, for every $x \in F$ we have $\bar{f}^{-1}(x)=f^{-1}(x)$ (if $x \neq s$ ) or $\bar{f}^{-1}(x)=f^{-1}(x) \cup Y_{S}$ (if $x=s$ ). Since the latter is a clopen set, continuity follows. Also, $\bar{f}$ satisfies CDC: the relevant case is when $d \cap \uparrow \bar{f}(w) \neq \emptyset$ for $d \in D, w \in Y_{S}$. We have $\bar{f}(w)=s$. But $f_{w}=S$ (because $w \in Y_{S}$ ), i.e. $f(Y \cap \uparrow w)=S$. Thus, there is $w^{\prime} \in Y$ with $w R^{+} w^{\prime}$ and $f\left(w^{\prime}\right)=s$. Since $w \notin Y$ and $w^{\prime} \in Y$, we have $w R w^{\prime}$. We can use the fact that $f$ satisfies the CDC: since $w^{\prime} \in Y=\operatorname{dom}(f)$ and $\uparrow \bar{f}(w)=\uparrow s=\uparrow f\left(w^{\prime}\right)$, we get $\uparrow f\left(w^{\prime}\right) \cap d \neq \emptyset$ and also $f\left(\uparrow w^{\prime}\right) \cap d \neq \emptyset$; as a consequence $\bar{f}(\uparrow w) \cap d$ is also not empty.

Case (II): $S$ does not have a reflexive root. We further distinguish two subcases, depending whether there are irreflexive $R^{+}$-quasi-maximal points in $Y_{S}$ or not. Notice that such points form the clopen antichain $Y_{S}^{\bullet}=Y_{S} \backslash \downarrow Y_{S}$.

Subcase (II.1): suppose $Y_{S}^{\bullet}=\emptyset$, i.e. there are no irreflexive quasi-maximal points in $Y_{S}$. Then, as noted above, every point in $Y_{S}$ can see a quasimaximal reflexive point in it. We put:

$$
\bar{f}(w)= \begin{cases}f(w), & \text { if } w \in Y, \\ s^{\circ}, & \text { if } w \in Y_{S} .\end{cases}
$$

It is easy to see that $\bar{f}$ is stable $\left(s^{\circ}\right.$ is reflexive). Now we also show that $\bar{f}$ is continuous. Indeed, for every $x \in F$ we have $\bar{f}^{-1}(x)=f^{-1}(x)$ (if $x \neq s^{\circ}$ ) or $\bar{f}^{-1}(x)=f^{-1}(x) \cup Y_{S}$ (if $\left.x=s^{\circ}\right)$. Since the latter is a clopen set, continuity follows. Also, $\bar{f}$ satisfies CDC: the relevant case is when $d \cap \uparrow \bar{f}(w) \neq \emptyset$ for $d \in D, w \in Y_{S}$. We have $\bar{f}(w)=s^{\circ}$ by construction and $d \cap\left(\left\{s^{\circ}\right\} \cup \uparrow S\right) \neq \emptyset$ by the choice of $s^{\circ}$. That is, either (i) $s^{\circ} \in d$ or (ii) there is an $s^{\prime} \in S$ such that $d \cap \uparrow s^{\prime} \neq \emptyset$. In case (i), we pick a quasi-maximal reflexive $w^{\prime}$ in $Y_{S}$ such that $w R^{+} w^{\prime}$ : since $\bar{f}\left(w^{\prime}\right)=s^{\circ}$, we have that $\bar{f}(\uparrow w) \cap d$ contains $s^{\circ}$ and is not empty. In case (ii), recall that $f_{w}=S$ (because $\left.w \in Y_{S}\right)$, i.e. $f(Y \cap \uparrow w)=S$. So there is $w^{\prime}$ such that $w R^{+} w^{\prime}, w^{\prime} \in Y$ and $f\left(w^{\prime}\right)=s^{\prime}$. Since $w \notin Y$, we must have $w R w^{\prime}$. We can then apply CDC for $f$ to get $w^{\prime \prime}$ such that $w^{\prime} R w^{\prime \prime}$ with $f\left(w^{\prime \prime}\right) \in d$. Thus, $w R w^{\prime \prime}$ by transitivity and $\bar{f}\left(w^{\prime \prime}\right)=f\left(w^{\prime \prime}\right) \in d$ : again, $\bar{f}(\uparrow w) \cap d$ is not empty.

Subcase (II.2): $Y_{S}^{\bullet}$ is not empty, i.e. $Y_{S}$ has irreflexive quasi-maximal points. Here we first make a preliminary extension $f_{0}$ of $f$ to $Y \cup Y_{S}^{\bullet}$ in 
order to include such points into the domain of the map. We then extend the new $f_{0}$ to $\bar{f}$ on $Y_{S} \backslash \downarrow Y_{S}^{\bullet}$.

Notice that $s^{\bullet} \notin S$ because $S \subseteq \uparrow s^{\bullet}$ and $S$ does not have a reflexive root. We put:

$$
f_{0}(w)= \begin{cases}f(w), & \text { if } w \in Y, \\ s^{\bullet}, & \text { if } w \in Y_{S}^{\bullet} .\end{cases}
$$

It is easy to see that $f_{0}$ is stable (points in $Y_{S}^{\bullet}$ are irreflexive). Now we also show that $f_{0}$ is continuous. Indeed, for every $x \in F$ we have $\left(f_{0}\right)^{-1}(x)=$ $f^{-1}(x)$ (if $x \neq s^{\bullet}$ ) or $\left(f_{0}\right)^{-1}(x)=f^{-1}(x) \cup Y_{S}^{\bullet}$ (if $x=s^{\bullet}$ ). Since the latter is a clopen set, continuity follows. Also, $f_{0}$ satisfies CDC: the relevant case is when $d \cap \uparrow f_{0}(w) \neq \emptyset$ for $d \in D, w \in Y_{S}^{\bullet}$. We have $f_{0}(w)=s^{\bullet}$ by construction. From $d \cap \uparrow s^{\bullet} \neq \emptyset$ we get that there is an $s^{\prime} \in d \cap \uparrow S$, i. e. there is $s^{\prime \prime} \in S$ such that $s^{\prime \prime} R s^{\prime} \in d$. Then, since $S=f_{w}=f(Y \cap \uparrow w)$, there is $w^{\prime \prime}$ such that $w^{\prime \prime} \in Y, w R^{+} w^{\prime \prime}$ and $f\left(w^{\prime \prime}\right)=s^{\prime \prime}$. Since $w \notin Y$, in fact we have $w R w^{\prime \prime}$ and by the CDC for $f\left(w^{\prime \prime} \in \operatorname{dom}(f)=Y\right)$, since $f\left(w^{\prime \prime}\right)=s^{\prime \prime} R s^{\prime} \in d$, we get $f\left(\uparrow w^{\prime \prime}\right) \cap d \neq \emptyset$. Thus $\uparrow f_{0}(w) \cap d \supseteq \uparrow f_{0}\left(w^{\prime \prime}\right) \cap d=\uparrow f\left(w^{\prime \prime}\right) \cap d \neq \emptyset$.

If we compute $Y_{S}$ with $f_{0}$ instead of $f$, we now get $Y_{S}^{\prime}=Y_{S} \backslash \downarrow Y_{S}^{\bullet}$ instead of $Y_{S}$ : since $s^{\bullet} \notin S$, for any $w$ we will have $f_{0}\left(\left(Y \cup Y_{S}^{\bullet}\right) \cap \uparrow w\right)=S$ if and only if $f(Y \cap \uparrow w)=S$ and $\uparrow w \cap Y_{S}^{\bullet}=\emptyset$, i.e. $w \in Y_{S} \backslash \downarrow Y_{S}^{\bullet}$. It follows that quasi-maximal points in $Y_{S}^{\prime}$ are all reflexive. We then can continue as in Subcase (II.1) above and get an extension $\bar{f}$.

So we extended $f$ to $\bar{f}$. We need to show that by repeating this procedure we cover the whole of $W$. This holds since the following is true: if some $S \subseteq F$ has been used for further extension of the map according to the above procedure, then this same $S$ can never occur again during any subsequent extensions.

Indeed let $f^{k}$, resp. $f^{n}$ be any further extensions of $f$ to $Y^{k}$, resp. $Y^{n}$, $k<n$. Suppose we have used some $S$ for $f^{k}$; then it cannot happen that $S$ can be also used for $f^{n}$.

Suppose, to the contrary, that $S$ occurs as one of the candidates to build $f^{n}$. Then in particular $S=f_{w}^{n-1}$ for some $w \in Y^{n} \backslash Y^{n-1}$. Then also $w \notin Y^{k}$, so $S \neq f_{w}^{k-1}$ (since $Y^{k}=Y^{k-1} \cup Y_{S}^{k-1}$ and $Y_{S}^{k-1}$ consists precisely of those $v \notin Y^{k-1}$ for which $f_{v}^{k-1}=S$ ). In fact by minimality of $S, f_{w}^{k-1}$ cannot be included in $S$, so $f_{w}^{k-1} \backslash S$ is nonempty. Now note that since $f^{n-1}$ is an extension of $f^{k-1}$, one has $f_{w}^{n-1} \supseteq f_{w}^{k-1}$, hence also $f_{w}^{n-1} \backslash S$ is nonempty, which contradicts the equality $S=f_{w}^{n-1}$ above.

It thus follows that after each next extension at least one subset of $F$ is excluded from all subsequent extension steps. Thus after some step $n$ there 
will be no $w \notin Y^{n}$ and no $S$ left with the property $f_{w}^{n}=S$. Which just means that there is no $w$ outside $Y^{n}$, i.e. $Y^{n}=W$.

$(1) \Rightarrow(4)$ : The proof is exactly the same as in Theorem 3.4.

Corollary 5.6. A canonical rule $\rho(F, D)$ has the following dichotomy property: it is either admissible or equivalent to an assumption-free rule.

Corollary 5.7. Admissibility is decidable for $\mathbf{K} 4$.

Proof. The proof is similar to the proof of Corollary 3.7.

Corollary 5.8. The rules $\left\{S_{n}^{\ell, m}: m, n \in \omega\right\} \cup\left\{T_{n}^{m}: m \in \omega\right\}$ form an admissible basis for $\mathbf{K} \mathbf{4}$.

Proof. The proof is similar to the proof of Corollary 3.8.

To conclude, we mention that the above results also hold for $\mathbf{S 4}$, with the following modifications: (i) rules $\left(T_{n}^{m}\right)$ should be removed from the admissible basis; (ii) rules $\left(S_{n}^{\ell, m}\right)$ are kept, but can be simplified (we do not need the parameter $\ell$ either, because the conjuncts $\bigwedge_{l=1}^{\ell}\left(\square v_{l} \rightarrow v_{l}\right)$ are now valid formulas); (iii) in Definition 5.3, conditions (3)-(4) are removed (thus a stable canonical rule is trivial in the new $\mathbf{S} \mathbf{4}$ sense iff it was just trivial ${ }^{\circ}$ in the old sense).

Remark 5.9. It is an open question whether the techniques developed in this paper would adapt well to fragments of IPC (or modal logics) and subreducts of Heyting algebras (or modal algebras). The implication and implicationconjunction-negation fragments of IPC are structurally complete, but not the implication-negation fragment (admissibility for the latter fragment is axiomatized in [7], see [20] for the positive fragment). Explicit axiomatizations for the admissible rules of the implication-disjunction fragment of IPC and pseudo-complemented distributive lattices are still lacking, however.

Remark 5.10. Recall that an algebra $P$ in a variety $\mathcal{V}$ is called projective if for any surjective homomorphism $p: A \rightarrow B$ of $\mathcal{V}$-algebras and any homomorphism $b: P \rightarrow B$ in the diagram

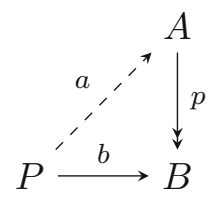

there exists a lift, i.e. a homomorphism $a: P \rightarrow A$ with $b=p a$. It is well known that free algebras are projective, that a retract of a projective algebra is projective, and that an algebra is projective if and only if it is a retract of a free algebra. 
For modal and Heyting algebras we can generalise the notion of projectivity to $D$-projectivity. We will discuss only the modal K4-case here. Let $(P, D)$ be a pair where $P$ is a $\mathbf{K} 4$-algebra and $D \subseteq P$. For brevity, let us call a map $h: P \rightarrow A$ a $D$-morphism if $h$ is a stable homomorphism satisfying CDC for $D$. We will denote $D$-morphisms by $h: P \rightsquigarrow A$.

For a subset $D \subseteq P$ of a K4-algebra $P$ we will call the algebra $P D$ projective if any diagram

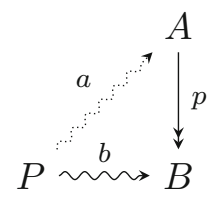

of K4-algebras has a $D$-lift, that is, for any surjective modal homomorphism $p$ and any $D$-morphism $b$ there is a $D$-morphism $a$ with $p a=b$. It can be shown that $P$ is $D$-projective if and only if it is a $D$-retract of a free K4algebra. The latter means that there exists a modal homomorphism $p: F \rightarrow$ $P$ from a free K4-algebra to $P$ and a $D$-morphism $f: P \rightsquigarrow F$ with $p f=i d_{P}$.

Then our main theorem 5.4 is nothing but a characterisation of finite $D$-projective K4-algebras. Namely it follows from the main theorem that for a finite K4-algebra $P$ and $D \subseteq P$, TFAE: (1) $P$ is $D$-projective, (2) $\rho(P, D)$ is not admissible, (3) The dual of $P$ satisfies the triviality conditions of Definition 5.3. Thus, in terms of $D$-projectivity we have the following dichotomy property: for any finite $\mathbf{K} 4$-algebra $P$ and any subset $D \subseteq P$, the stable canonical rule $\rho(P, D)$ is not admissible if and only if $P$ is $D$ projective.

Remark 5.11. Admissibility and unification over the basic (non-transitive) modal logic $\mathbf{K}$ are long-standing open problems. Although the proofs of this paper do not apply to $\mathbf{K}$ directly, we note that unlike the canonical rules of [18], stable canonical rules axiomatize consequence relations over $\mathbf{K}$. It remains open whether stable canonical rules could be applicable in analysing admissibility for non-transitive logics: in particular, whether they could be used in obtaining some dichotomy property for $\mathbf{K}$.

Acknowledgements. The authors are very grateful to Guram Bezhanishvili for many interesting discussions and his encouragement of this project.

Open Access. This article is distributed under the terms of the Creative Commons Attribution 4.0 International License (http://creativecommons. 
org/licenses/by/4.0/), which permits unrestricted use, distribution, and reproduction in any medium, provided you give appropriate credit to the original author(s) and the source, provide a link to the Creative Commons license, and indicate if changes were made.

\section{References}

[1] Bezhanishvili, G., N. Bezhanishvili, and R. Iemhoff, Stable canonical rules, Journal of Symbolic Logic, to appear. Available as the ILLC Prepublication Series Report PP-2014-08.

[2] Bezhanishvili, G., Y. Bezhanishvili, and J. Ilin, Cofinal stable logics. Submitted. Available as the ILLC Prepublication Series Report PP-2015-08, 2015.

[3] Bezhanishvili, G., R. Mines, and P. J. Morandi, Topo-canonical completions of closure algebras and Heyting algebras, Algebra Universalis 58(1):1-34, 2008.

[4] Bezhanishvili, N., and S. Ghilardi, Multiple-conclusion rules, hypersequents syntax and step frames, in R. Gore, B. Kooi, and A. Kurucz, (eds.), Advances in Modal Logic (AiML 2014), An extended version available as ILLC Prepublication Series Report PP-2014-05, College Publications, 2014, pp. 54-61.

[5] Blackburn, P., M. De Rijke, and Y. Venema, Modal Logic, Cambridge University Press, Cambridge, 2001.

[6] Chagrov, A., and M. Zakharyaschev, Modal Logic, The Clarendon Press, Oxford, 1997.

[7] Cintula, P., and G. Metcalfe, Admissible rules in the implication-negation fragment of intuitionistic logic, Annals of Pure and Applied Logic 162(2):162-171, 2010.

[8] Davey, B. A., and H. A. Priestley, Introduction to Lattices and Order, 2nd edn., Cambridge University Press, New York, 2002.

[9] Esakia, L., Topological Kripke models, Soviet Mathematics Doklady 15:147-151, 1974.

[10] Friedman, H., One hundred and two problems in mathematical logic, Journal of Symbolic Logic 40:113-129, 1975.

[11] Ghilardi, S., Unification in intuitionistic logic, Journal of Symbolic Logic 64(2):859880, 1999.

[12] Ghilardi, S., Best solving modal equations, Annals of Pure and Applied Logic 102(3):183-198, 2000.

[13] Ghilardi, S., Continuity, freeness, and filtrations, Journal of Applied Non-classical Logics 20(3):193-217, 2010.

[14] Goudsmit, J., Intuitionistic rules, admissible rules of intermediate logics. PhD thesis, University of Utrecht, 2015.

[15] IEmhoff, R., On the admissible rules of intuitionistic propositional logic, Journal of Symbolic Logic 66(1):281-294, 2001.

[16] Iemhoff, R., Consequence relations and admissible rules, Journal of Philosophical Logic 2015. doi:10.1007/s10992-015-9380-8.

[17] JeřÁBeK, E., Independent bases of admissible rules, Logic Journal of IGPL 16(3):249$267,2008$.

[18] Jeř́́Bek, E., Canonical rules, Journal of Symbolic Logic 74(4):1171-1205, 2009. 
[19] Kracht, M., Tools and Techniques in Modal Logic, Studies in Logic and the Foundations of Mathematics, vol. 142, North-Holland Publishing Co., Amsterdam, 1999.

[20] Odintsov, S., and V. Rybakov, Unification and admissible rules for paraconsistent minimal Johanssons' logic $\mathbf{J}$ and positive intuitionistic logic IPC+, Annals of Pure and Applied Logic 164(7-8):771-784, 2013.

[21] Rasiowa, H., and R. Sikorski, The Mathematics of Metamathematics. Monografie Matematyczne, Tom 41. Państwowe Wydawnictwo Naukowe, Warsaw, 1963.

[22] Rozière, P., Regles admissibles en calcul propositionnel intuitionniste. PhD thesis, Université Paris VII, 1992.

[23] Rybakov, V. V., Bases of admissible rules of the logics S4 and Int, Algebra i Logika 24(1):87-107, 123, 1985.

[24] Rybakov, V. V., Admissibility of Logical Inference Rules, Studies in Logic and the Foundations of Mathematics, vol. 136, North-Holland Publishing Co., Amsterdam, 1997.

[25] Rybakov, V. V., Construction of an explicit basis for rules admissible in modal system S4, Mathematical Logic Quarterly 47(4):441-446, 2001.

[26] Rybakov, V. V. Problems of substitution and admissibility in the modal system grz and in intuitionistic propositional calculus, Annals of Pure and Applied Logic 50:71106, 1990.

[27] Rybakov, V. V., Rules of inference with parameters for intuitionistic logic, Journal of Symbolic Logic 57:33-52, 1992.

[28] Venema, Y., Algebras and coalgebras. In P. Blackburn, J. van Benthem, and F. Wolter (eds.), Handbook of Modal Logic, Studies in Logic and Practical Reasoning, vol. 3. Elsevier, New York, 2007, pp. 331-426.

[29] Zakharyaschev, M., Canonical formulas for K4 I. Basic results, Journal of Symbolic Logic 57(4):1377-1402, 1992.

\section{N. BeZhANishviLi}

Institute for Logic, Language and Computation, University of Amsterdam, P.O. Box 94242, 1090 GE Amsterdam, The Netherlands

N.Bezhanishvili@uva.nl

D. Gabelaia, M. Jibladze

Department of Mathematical Logic, TSU Razmadze Mathematical Institute, 6 Tamarashvili Str., 0177 Tbilisi, Georgia

gabelaia@gmail.com

M. JiBLADZE

mamuka.jibladze@gmail.com

\section{S. GHILARDI}

Dipartimento di Matematica, Università degli Studi di Milano via C. Saldini 50, 20133 Milano, Italy silvio.ghilardi@unimi.it 